\title{
Miniaturized protein offers treatment hopes for Rett syndrome
}

The restoration of two key domains in methyl-CpG-binding protein 2 (MECP2) could be sufficient to treat Rett syndrome, according to new research in mice. The study opens new avenues for development of a gene therapy for the condition.

Rett syndrome is a rare neurological disorder that disrupts cognition, movement and growth. The condition is primarily diagnosed in girls aged 6-18 months and is usually caused by loss-of-function mutations in the $\mathrm{X}$ chromosomal gene $M E C P 2$, which encodes a multifunctional protein that acts as a hub for modulators of gene expression.

Owing to the multitude of proteins bound by MECP2, characterization of the mechanisms that underlie Rett syndrome has been challenging. Symptoms were assumed to be irreversible as MECP2 mutations were thought to disrupt brain development; however, research in animal models has suggested that restoration of MECP2 function can ameliorate brain dysfunction and so could present a viable therapy.

MECP2 mutations are concentrated in two locations: the methylCpG-binding domain (MBD), which binds methylated DNA, and the nuclear receptor corepressor interaction domain (NID), which binds co-repressors of gene expression. In the new study, researchers generated several transgenic mouse lines that express shortened versions of MECP2 to determine whether restoration of the MBD and NID

Mice

expressing

$\triangle \mathrm{N}$ or $\triangle \mathrm{NC}$ showed no phenotypes

for over a year, whereas MECP2-null mice had a median survival of 9 weeks domains alone could be used as a therapy.

The team found that expression of truncated MECP2 containing the MBD and NID domains but missing the amino terminus $(\Delta \mathrm{N})$ or both the amino and carboxyl termini $(\triangle \mathrm{NC})$ almost completely prevented the deficits in cognition, coordination and lifespan that are shown by MECP2-null mice. Mice expressing $\triangle \mathrm{N}$ or $\triangle \mathrm{NC}$ showed no phenotypes for over a year, whereas MECP2-null mice had a median survival of 9 weeks. The researchers also generated a miniaturized MECP2 lacking the amino acids between the MBD and NID domains as well as both termini of the protein ( $\triangle \mathrm{NIC}$ ); animals expressing $\Delta$ NIC exhibited only mild symptoms after 1 year.

The short length of the $\triangle$ NIC gene enabled the team to incorporate it into an adeno-associated virus. Injection of this virus into the brains of MECP2-null mice reduced the severity of symptoms and significantly increased survival compared with vehicle-injected control mice. The result shows that this strategy could present a candidate for a future gene therapy.

"Despite evolutionary conservation of the entire MECP2 protein sequence, the DNA and co-repressor binding domains alone are sufficient to avoid Rett syndrome-like defects and may, therefore, have therapeutic utility," the authors conclude in their new paper.

Charlotte Ridler

ORIGINAL ARTICLE Tillotson, R. et al. Radically truncated $\mathrm{MeCP} 2$ rescues Rett syndrome-like neurological defects. Nature 550, 398-401 (2017) FURTHER READING Leonard, H. et al. Clinical and biological progress over 50 years in Rett syndrome. Nat. Rev. Neurol. 13, 37-51 (2017) 